Pacific Journal of Mathematic 


\section{A COMMON FIXED POINT THEOREM FOR A COMMUTING FAMILY OF NONEXPANSIVE MAPPINGS}

Ronald E. BruCK, JR.

It is shown that if a closed convex subset $C$ of a Banach space has both the fixed point property and the conditional fixed point property for nonexpansive mappings and $C$ is either weakly compact or bounded and separable, then any commuting family of nonexpansive self-mappings of $C$ has a common fixed point. The set of common fixed points is a nonexpansive retract of $C$.

Introduction. Let $E$ be a real or complex Banach space and $C$ a nonempty closed convex subset of $E$. Our purpose is to prove the following generalization of the DeMarr-Browder-Belluce-Kirk-Lim [8, $4,1,2,15]$ fixed point theorem:

THEOREM 1. Suppose $C$ has both the fixed point property and the conditional fixed point property for nonexpansive mappings, and $C$ is either weakly compact or bounded and separable. Then for any commuting family $S$ of nonexpansive self-mappings of $C$, the set $F(S)$ of common fixed points of $S$ is a nonempty nonexpansive retract of $C$.

(A mapping $f: C \rightarrow E$ is nonexpansive if $\|f(x)-f(y)\| \leqq\|x-y\|$ for all $x, y \in C ; C$ has the fixed point property for nonexpansive mappings (abbreviation: FPP) if every nonexpansive $f: C \rightarrow C$ has a fixed point; $C$ has the hereditary fixed point property for nonexpansive mappings (abbreviation: HFPP) if every nonempty bounded closed convex subset of $C$ has the FPP; finally, $C$ has the conditional fixed point property for nonexpansive mappings (abbreviation: $C F P P$ ) if every nonexpansive $f: C \rightarrow C$ satisfies

either $f$ has no fixed points in $C$, or $f$ has a fixed point in $(C F P)$ : every nonempty bounded closed convex $f$-invariant subset of $C$.

This condition was introduced in [6]. A subset $F$ of $C$ is a nonexpansive retract of $C$ if either $F=\varnothing$ or there exists a retraction of $C$ onto $F$ which is a nonexpansive mapping; this was introduced in $[5,7]$. For the definition of normal structure see Brodskii-Milman [3], Kirk [12], or Belluce and Kirk [1].)

The existence of a common fixed point was established by DeMarr 
[8] when $C$ is compact, by Belluce and Kirk [1] when $C$ is weakly compact and has normal structure and $S$ is finite, by Belluce and Kirk [2] when $C$ is weakly compact and has complete normal structure, by Browder [4] when $E$ is uniformly convex and $C$ is bounded, by Bruck [6] when $C$ is weakly compact and has the HFPP and $S$ is finite, and finally by $\operatorname{Lim}[15]$ when $C$ is weakly compact and has normal structure. The principal difficulty in the noncompact case has been proving the theorem for infinite families. In the compact case, on the other hand, the requirement that $S$ be commutative has been relaxed to the assumption that $S$ be a left reversible semigroup. See Takahashi [17], Mitchell [16], Holmes and Lau [9, 10].

Our approach to Theorem 1 is very different from that of these references (except [6]) in that we completely avoid the use of normal structure. The increase in generality is slight (normal structure surely suffices for any applications) but we feel that our proof cuts closer to the geometric structure which underlies Theorem 1. The key to that structure is:

THEOREM 2. Suppose $f: C \rightarrow C$ is nonexpansive and satisfies $(C F P)$, and $C$ is either locally weakly compact or separable. Then $F(f)$, the fixed point set of $f$, is a nonexpansive retract of $C$.

Theorem 2 was proven in [6] for the case when $C$ is locally weakly compact (i.e., every bounded closed convex subset of $C$ is weakly compact). An earlier version was announced in [5].

We shall prove Theorem 2 from the more general:

THEOREM 3. Let $X$ be a Hausdorff topological space and $S$ a semigroup of mappings on $X$. Suppose that either (a) $S$ is compact in the topology of pointwise convergence or (b) $X$ is a separable complete metric space and $S$ is equicontinuous. Then there exists in $\bar{S}$ a retraction of $X$ onto $F(S)$ iff the following condition is satisfied:

$(F P): \quad$ each nonempty closed $S$-invariant subset of $X$ contains a
fixed point of $S$, or equivalently,

$(F P)^{\prime}:$ whenever $x \in X$ then $\mathrm{Cl}(S x)$ contains a fixed point of $S$.

( $\bar{S}$ denotes the closure of $S$ in $X^{x}$ in the topology of pointwise convergence; a fixed point of $S$ is a point $x$ such that $s(x)=x$ for all $s \in S$; Cl denotes closure; and the set of fixed points of $S$ is denoted by $F(S)$.) 
1. Proofs. First we prove Theorem 1 from Theorem 2 and a sequence of lemmas; then we prove Theorem 2 from Theorem 3; finally, we prove Theorem 3.

The crucial result which permits the extension of [6, Theorem 7] to infinite families is:

Lemma 1. If $C$ is bounded and $\left\{F_{n}\right\}$ is a descending sequence of nonempty nonexpansive retracts of $C$, then $\bigcap_{n} F_{n}$ is the fixedpoint set of some nonexpansive $r: C \rightarrow C$.

Proof. For each $n$ choose a nonexpansive retraction $r_{n}$ of $C$ onto $F_{n}$. Choose a sequence $\left\{\lambda_{n}\right\}$ with $0<\lambda_{n}, \sum \lambda_{n}=1$, and

$$
\lim _{n} \sum_{j=n+1}^{\infty} \lambda_{j} / \sum_{j=n}^{\infty} \lambda_{j}=0
$$

(For example, we may take $\lambda_{n}=1 / n !-1 /(n+1)$ ! for $n=1,2, \cdots$.) Put $r=\sum \lambda_{n} r_{n}$.

Now it is obvious that we have defined a nonexpansive mapping $r: C \rightarrow C$ with $\bigcap_{n} F_{n} \subset F(r)$. To prove the reverse inclusion, let $x$ be a fixed point of $r$. Then

$$
\begin{aligned}
& \left\|x-r_{n}(x)\right\|=\left\|r(x)-r_{n}(x)\right\| \\
& \quad=\left\|\sum_{j=1}^{\infty} \lambda_{j}\left[r_{j}(x)-r_{n}(x)\right]\right\| \leqq \sum_{j=1}^{\infty} \lambda_{j}\left\|r_{j}(x)-r_{n}(x)\right\| .
\end{aligned}
$$

Now for $1 \leqq j<n, r_{n}(x) \in F_{n} \subset F_{j}$ so $r_{j} r_{n}(x)=r_{n}(x)$ and

$$
\left\|r_{j}(x)-r_{n}(x)\right\|=\left\|r_{j}(x)-r_{j} r_{n}(x)\right\| \leqq\left\|x-r_{n}(x)\right\| ;
$$

for $j=n,\left\|r_{j}(x)-r_{n}(x)\right\|=0$; finally, for $j>n,\left\|r_{j}(x)-r_{n}(x)\right\| \leqq d$, the diameter of $C$. Thus (2) implies

$$
\left\|x-r_{n}(x)\right\| \leqq \sum_{j=1}^{n-1} \lambda_{j}\left\|x-r_{n}(x)\right\|+d \sum_{j=n+1}^{\infty} \lambda_{j} .
$$

Since $\sum \lambda_{j}=1$, this in turn implies

$$
\left\|x-r_{n}(x)\right\| \leqq d \sum_{j=n+1}^{\infty} \lambda_{j} / \sum_{j=n}^{\infty} \lambda_{j}
$$

By (1), therefore, $r_{n}(x) \rightarrow x$ strongly as $n \rightarrow \infty$. But $\left\{F_{n}\right\}$ is descending, hence $r_{n}(x) \in F_{m}$ for $n \geqq m$; and $F_{m}$ is strongly closed because it is the fixed point set of the continuous mapping $r_{m}$. Therefore, $\lim _{n} r_{n}(x)=x$ belongs to $F_{m}$ for $m=1,2, \cdots$, so that $F(r) \subset \bigcap_{n} F_{n}$.

Our approach to Theorem 1 is through intersections of nonexpansive retracts. 
Lemma 2. Suppose $C$ is bounded, separable, and has both the FPP and the CFPP. Then for any family $\mathscr{F}$ of nonempty nonexpansive retracts of $C$ which is directed by $\supset, H=\bigcap\{F \mid F \in \mathscr{F}\}$ is a nonempty nonexpansive retract of $C$.

Proof of Lemma 2 from Lemma 1 and Theorem 2. There is a countable subfamily $\mathscr{F}^{\prime}$ of $\mathscr{F}$ such that $H=\bigcap\left\{F \mid F \in \mathscr{F}^{\prime}\right\}$ (otherwise $\{C \backslash F \mid F \in \mathscr{F}\}$ is an open cover of $C \backslash H$ with no countable subcover, which is impossible because $C \backslash H$ is a separable metric space). Using the fact that $\mathscr{F}$ is directed by $\supset$ we can therefore find a descending sequence $\left\{F_{n}\right\}$ in $\mathscr{F}$ with $\bigcap_{n} F_{n}=H$. By Lemma 1, $H=F(r)$ for some nonexpansive $r: C \rightarrow C$; since $C$ has the $C F P P$, Theorem 2 implies $H$ is a nonexpansive retract of $C$; finally, since $C$ has the $F P P, H \neq \varnothing$.

Lemma 2 is much more difficult to prove when $C$ is weakly compact instead of separable.

LeMma 3. Lemma 2 remains valid if $C$ is weakly compact instead of bounded and separable.

Proof of Lemma 3 from Lemma 2 and Theorem 3. Define

$$
\begin{gathered}
S=\{s: C \rightarrow C \mid s \text { is nonexpansive and } H \subset F(s)\}, \\
\widetilde{S}=\{s \in S \mid F \subset F(s) \text { for some } F \in \mathscr{F}\} .
\end{gathered}
$$

Both $S$ and $\widetilde{S}$ are convex semigroups on $C$ : If $0 \leqq \lambda \leqq 1$ and $s_{1}, s_{2}$ belong to $S$ (resp. $\widetilde{S}$ ), then $s_{1} s_{2}$ and $\lambda s_{1}+(1-\lambda) s_{2}$ belong to $S$ (resp. $\widetilde{S})$. (For $\widetilde{S}$ this uses the fact that $\mathscr{F}$ is directed by $\supset$.) We shall show that $F(S)=H, S$ is compact in the topology of weak pointwise convergence, and $S$ satisfies $(F P)^{\prime}$. When this is done, Theorem 3 implies the existence of a retraction $e \in \bar{S}$ of $C$ onto $H$ (which is therefore nonempty). But since $S$ is compact, $\bar{S}=S$, so $e$ is a nonexpansive retraction of $C$ onto $H$.

Now it is clear from the definition of $S$ and $\widetilde{S}$ that $H \subset F(S) \subset$ $F(\widetilde{S})$. Suppose $x \in F(\widetilde{S})$. For each $F \in \mathscr{F}$ choose a nonexpansive retraction $r_{F}$ of $C$ onto $F$; then $r_{F} \in \widetilde{S}$, hence $r_{F}(x)=x$, hence $x \in F$, for each $F \in \mathscr{F}$. That is, $F(\widetilde{S}) \subset H$, so $H=F(S)=F(\widetilde{S})$.

Give $C$ the weak topology, so it is compact. By Tychonoff's theorem $C^{C}$ is compact. But it is clear from the weak lower semicontinuity of the norm that $S$ is closed in $C^{C}$, hence compact in the topology of (weak) pointwise convergence.

We finally come to the most difficult verification: That $S$ has a fixed point in each $\mathrm{Cl}(S x)$. Now $S x=\{s(x) \mid s \in S\}$ is convex (because 
$S$ is convex), $S$-invariant (because $S$ is a semigroup), and bounded (because $S x \subset C$ and $C$ is bounded). Therefore, $\mathrm{Cl}(S x)=$ weak-Cl $(S x)=$ strong-Cl $(S x)$ is nonempty, strongly closed, convex, and $S$-invariant. Since $\widetilde{S} \subset S, \mathrm{Cl}(S x)$ is also $\widetilde{S}$-invariant, so Zorn's lemma and the weak compactness of closed convex subsets of $C$ imply the existence of a minimal nonempty closed convex $\widetilde{S}$-invariant subset $K$ of $\mathrm{Cl}(S x)$. We shall show that $K$ consists of a single point $y^{*}$, which must be a fixed point of $\widetilde{S}$ (and hence of $S$ ) because $K$ is $\widetilde{S}$-invariant. The proof of the lemma will then be complete.

It is convenient to introduce three definitions. First, if $S^{\prime} \subset \widetilde{S}$ and $M$ is a nonempty closed convex subset of $K$, the $S^{\prime}$-extension of $M$ is the smallest closed convex $S^{\prime}$-invariant subset of $K$ which contains $M$.

Second, if $S^{\prime} \subset \widetilde{S}$ then $S^{\prime}$ is augmented provided for each $s \in S^{\prime}$ there is at least one $F \in \mathscr{F}$ such that $r_{F} \in S^{\prime}$ and $F \subset F(s)$, and $\left\{F \in \mathscr{F} \mid r_{F} \in S^{\prime}\right\}$ is directed by $\supset$.

Third, a subset $S^{\prime}$ of $\widetilde{S}$ is almost transitive on a subset $D$ of $K$ if for each $p, q$ in $D$ there exists a sequence $\left\{s_{n}\right\} \subset S^{\prime}$ with $s_{n}(p) \rightarrow$ $q$ strongly.

We make three important remarks on these definitions. First, if $M$ is a separable closed convex subset of $K$ and $S^{\prime}$ is a countable subset of $\widetilde{S}$, then the $S^{\prime}$-extension of $M$ is also separable. Second, any countable subset of $\widetilde{S}$ is contained in a countable augmented subset of $\widetilde{S}$. These remarks are easy to verify. Finally, if $D$ is any countable subset of $K$ then there exists a countable subset of $\widetilde{S}$ which is almost transitive on $D$. To see this, first note that if $p \in K$ then $\mathrm{Cl}(\widetilde{S} p)=K$ because $\mathrm{Cl}(\widetilde{S} p)$ is a nonempty closed convex $\widetilde{S}$-invariant subset of $K$, and $K$ is minimal with respect to these properties. Because the strong and weak closures of $\widetilde{S} p$ coincide, given any $p, q$ in $D$ there exists $\left\{s_{n}\right\} \subset \widetilde{S}$ such that $s_{n}(p) \rightarrow q$ strongly. Taking the union of such sequences as $p$ and $q$ run through $D$ yields a countable subset of $S$ which is almost transitive on $D$.

Suppose, in order to reach a contradiction, that $K$ consists of more than one point. Then there exists a nontrivial closed line segment $K_{0}$ in $K$. Find a countable augmented subset $S_{0}$ of $\widetilde{S}$ which is almost transitive on $K_{0}$ and let $K_{1}$ be the $S_{0}$-extension of $K_{0}$. This is possible by our preceding remarks and $K_{1}$ is a separable, closed convex subset of $K$ with $K_{0} \subset K_{1}$.

In general, once a separable closed convex $K_{n}$ has been defined for some $n \geqq 1$, choose a countable dense subset $D_{n}$ of $K_{n}$ with $D_{n-1} \subset D_{n}$, and a countable augmented subset $S_{n}$ of $\widetilde{S}$ which is almost transitive on $D_{n}$, with $S_{n-1} \subset S_{n}$; then let $K_{n+1}$ be the $S_{n}$-extension of $K_{n}$. Thus $K_{n+1}$ is a separable closed convex subset of $K$ and $K_{n} \subset K_{n+1}$. 
Having defined the ascending sequences $\left\{K_{n}\right\},\left\{D_{n}\right\}$, and $\left\{S_{n}\right\}$, let $K^{*}=\mathrm{Cl}_{n} K_{n}, D^{*}=\bigcup_{n} D_{n}$, and $S^{*}=\bigcup_{n} S_{n}$. Obviously $K^{*}$ is a separable closed convex subset of $K, D^{*}$ is a countable dense subset of $K^{*}$, and $S^{*}$ is a countable augmented subset of $\widetilde{S}$ which is almost transitive on $D^{*}$. Since $\mathrm{U}_{n} K_{n}$ is $S^{*}$-invariant, so is $K^{*}$.

Define $\mathscr{F}^{*}=\left\{F \in \mathscr{F} \mid r_{F} \in S^{*}\right\}$ and $\mathscr{F}^{*} \cap K^{*}=\left\{F \cap K^{*} \mid F \in \mathscr{F}^{*}\right\}$. Since $K^{*}$ is $S^{*}$-invariant, for $F \in \mathscr{F}^{*}$ the restriction $\left.r_{F}\right|_{K^{*}}$ is a nonexpansive retraction of $K^{*}$ onto the (necessarily nonempty) set $F \cap K^{*}$. Thus $\mathscr{F}^{*} \cap K^{*}$ is a family of nonempty nonexpansive retracts of $K^{*}$ which is countable (because $S^{*}$ is countable) and directed by $\supset$ (because $S^{*}$ is augmented). It is tempting to apply Lemma 2 to conclude that $\bigcap\left\{F \cap K^{*} \mid F \in \mathscr{F}^{*}\right\}$ is nonempty, but while $K^{*}$ is separable we do not know that it has the FPP. However, the method of proof of Lemma 2 shows that $\bigcap\left\{F \cap K^{*} \mid F \in \mathscr{F}^{*}\right\}$ is the fixed point set of a nonexpansive mapping $f^{*}: K^{*} \rightarrow K^{*}$ defined as some convex linear combination

$$
f^{*}=\left.\sum_{F \in \mathscr{F} *} \lambda_{F} r_{F}\right|_{K^{*}}
$$

While $K^{*}$ may not have the $F^{\prime} P P$ in general, it does for this particular nonexpansive mapping because $f^{*}=\left.f\right|_{K^{*}}$, where

$$
f=\sum_{F \in \mathscr{F} *} \lambda_{F} r_{F},
$$

while $C$ has the FPP and the CFPP. Therefore, $F\left(f^{*}\right) \neq \varnothing$. But

$$
F\left(f^{*}\right)=\bigcap\left\{K^{*} \cap F \mid F \in \mathscr{F}^{*}\right\},
$$

hence there exists $y^{*} \in \bigcap\left\{K^{*} \cap F \mid r_{F} \in S^{*}\right\}$. Since $r_{F}\left(y^{*}\right)=y^{*}$ when $r_{F} \in S^{*}$ and $S^{*}$ is augmented, therefore $y^{*} \in F\left(S^{*}\right)$.

But $S^{*}$ is almost transitive on $D^{*}$; for any $p, q$ in $D^{*}$ there exists $\left\{s_{n}\right\} \subset S^{*}$ such that $s_{n}(p) \rightarrow q$. Since $y^{*} \in F\left(S^{*}\right), s_{n}\left(y^{*}\right)=y^{*}$ for all $n$, hence

$$
\left\|s_{n}(p)-y^{*}\right\|=\left\|s_{n}(p)-s_{n}\left(y^{*}\right)\right\| \leqq\left\|p-y^{*}\right\|,
$$

so in the limit $\left\|q-y^{*}\right\| \leqq\left\|p-y^{*}\right\|$. Of course the symmetric inequality also holds, so $\left\|q-y^{*}\right\|=\left\|p-y^{*}\right\|$ for all $p, q$ in $D^{*}$. But $D^{*}$ is dense in $K^{*}$, hence all points of $K^{*}$ are equidistant from $y^{*}$. Since $y^{*}$ itself is in $K^{*}$, all points in $K^{*}$ are at distance 0 from $y^{*}$, i.e., $K^{*}$ is a single point. This is a contradiction since $K_{0}$ is a nontrivial line segment and $K_{0} \subset K^{*}$.

We are not aware of any shorter proof of Lemma 3, although one is obviously desirable. 
Proof of Theorem 1 from Lemma 2, Lemma 3, and Theorem 2. First we shall show that if $s_{1}, \cdots, s_{n}$ are commuting nonexpansive self-mappings of $C$, then $\bigcap_{j=1}^{n} F\left(s_{j}\right)$ is a nonempty nonexpansive retract of $C$. The proof is by induction on $n$.

If $n=1$, then $F\left(s_{1}\right)$ is a nonexpansive retract of $C$ by Theorem 2 and the assumption that $C$ has the CFPP; $F\left(s_{1}\right) \neq \varnothing$ by the assumption that $C$ has the FPP.

Now suppose $\bigcap_{j=1}^{n} F\left(s_{j}\right)$ is a nonempty nonexpansive retract of $C$ and $s_{n+1}$ commutes with $s_{1}, \cdots$, and $s_{n}$. Put $F_{n}=\bigcap_{j=1}^{n} F\left(s_{j}\right)$ and let $r$ be a nonexpansive retraction of $C$ onto $F_{n}$. We claim that $F\left(s_{n+1} \circ r\right)=\bigcap_{j=1}^{n+1} F\left(s_{j}\right)$. The inclusion $\bigcap_{j=1}^{n+1} F\left(s_{j}\right) \subset F\left(s_{n+1} r\right)$ is trivial; to prove the reverse inclusion, suppose $s_{n+1} r(x)=x$. Now $r(x) \in F_{n}$, and since $s_{n+1}$ commutes with $s_{1}, \cdots$, and $s_{n}, F_{n}$ is $s_{n+1}$-invariant; therefore, $s_{n+1} r(x) \in F_{n}$. But $x=s_{n+1} r(x)$, therefore $x \in F_{n}$. But then $r(x)=x$, so $x=s_{n+1} r(x)=s_{n+1}(x)$. We have shown $x \in F_{n} \cap F\left(s_{n+1}\right)$, so $F\left(s_{n+1} r\right)=\bigcap_{j=1}^{n+1} F\left(s_{j}\right)$.

The fixed-point set of a nonexpansive self-mapping of $C$ is, by Theorem 2 and the assumptions on $C$, a nonempty nonexpansive retract of $C$. Thus $\bigcap_{j=1}^{n+1} F\left(s_{j}\right)$ is a nonempty nonexpansive retract of $C$, which completes the induction.

Now let $\mathscr{F}$ be the family of the finite intersections of fixed point sets of mappings in the commutative family $S$. We have just shown that $\mathscr{F}$ is a family of nonempty nonexpansive retracts of $C$, and $\mathscr{F}$ is obviously directed by $\supset$. By Lemma 2 or Lemma 3, depending on whether $C$ is weakly compact or bounded and separable, $\bigcap\{F \mid F \in \mathscr{F}\}$ is a nonempty nonexpansive retract of $C$. But this intersection is obviously $F(S)$.

Proof of Theorem 2 from Theorem 3. We may suppose $F(f) \neq$ $\varnothing$. Put $S=\{s: C \rightarrow C \mid s$ is nonexpansive and $F(f) \subset F(s)\}$. We claim that $S$ is a semigroup on $C, F(S)=F(f)$, and $S$ satisfies $(F P)^{\prime}$.

Obviously $S$ is a semigroup and $F(f) \subset F(S)$; since $f \in S$ the reverse inclusion is also true.

For $x \in C, S x$ is clearly nonempty, convex, and $f$-invariant. If $y_{0} \in F(f)$ then $s\left(y_{0}\right)=y_{0}$, hence

$$
\left\|s(x)-y_{0}\right\|=\left\|s(x)-s\left(y_{0}\right)\right\| \leqq\left\|x-y_{0}\right\|,
$$

for all $s \in S$, therefore $S x$ is bounded. Since $f$ is continuous, $\mathrm{Cl}(S x)$ is a nonempty bounded closed convex $f$-invariant subset of $C$, and since $F(f) \neq \varnothing$ and $f$ satisfies $(C F P), f$ has a fixed point in $\mathrm{Cl}(S x)$. But $F(f)=F(S)$, therefore $S$ satisfies $(F P)^{\prime}$.

Theorem 2 has already been proven in [6] for the case when $C$ is locally weakly compact, so we may assume $C$ is separable in the metric topology induced by the norm. Then $C$ is a separable 
complete metric space and $S$ is equicontinuous, so Theorem 3 implies the existence of a retraction $e \in \bar{S}$ of $C$ onto $F(S)$. Since $\bar{S}=S$ and $F(S)=F(f), e$ is a nonexpansive retraction of $C$ onto $F(f)$.

Proof of necessity in Theorem 3. A retraction of $X$ onto $F(S)$ is simply a mapping $e: X \rightarrow X$ with range $(\mathrm{e})=F(S)$, for which $e^{2}=$ $e$. Continuity of $e$ is not required.

Suppose $e \in \bar{S}$ is a retraction of $X$ onto $F(S)$, and suppose $M$ is a nonempty closed $S$-invariant subset of $X$. Then $M$ is obviously invariant under $\bar{S}$, so $e(M) \subset M$. Since $e$ is a retraction onto $F(S)$, also $e(M) \subset F(S)$. Thus $F(S) \cap M$ contains at least the set $e(M)$ and is therefore nonempty, i.e., $(F P)$ is satisfied.

Proof of sufficiency in Theorem 3. Our strategy here is to show that $\bar{S}$ is a semigroup on $X$ and then to construct a oneelement left ideal $\{e\}$ of $\bar{S}$; for in that case $e$ must be a retraction of $X$ onto $F(S)$. To see this, observe that $F(e) \subset$ range $(e)$ (true of any mapping), range $(e) \subset F(\bar{S})$ (because $s e=e$ for all $s \in \bar{S}$ implies $e(x) \in F(\bar{S})$ for all $x \in X), F(\bar{S}) \subset F(e)$ (because $e \in \bar{S}$ ), and $F(\bar{S})=$ $F(S)$ (recall $X$ is Hausdorff). Thus range $(e)=F(e)=F(S)$, which implies $e$ is a retraction of $X$ onto $F(S)$.

$\bar{S}$ is a semigroup under hypothesis (a) because $\bar{S}=S$. On the other hand, under (b) composition is jointly continuous on $\bar{S} \times \bar{S}$ and since $S$ is a semigroup, $\bar{S}$ must also be a semigroup.

It is easier to construct a one-element left ideal of $\bar{S}$ under hypothesis (a), for by an elementary compactness-Zorn argument there must then exist a minimal closed left ideal $J$ of $S$. If $x_{0} \in X$ then $J x_{0}=\left\{j\left(x_{0}\right) \mid j \in J\right\}$ is compact (it is the image of the compact set $J$ in $S$ under the continuous projection $s \rightarrow s\left(x_{0}\right)$ of $S$ into $X$ ). $J x_{0}$ is $S$-invariant because $J$ is a left ideal of $S$; by condition $(F P)$, $J x_{0}$ must contain some fixed point $u_{0}$ of $S$. Define $I=\left\{j \in J \mid j\left(x_{0}\right)=u_{0}\right\}$. $I$ is nonempty because $u_{0} \in J x_{0} ; I$ is closed in $S$ (in the topology of pointwise convergence); and $I$ is a left ideal of $S$ (because $J$ is a left ideal and $u_{0} \in F(S)$ ). Since $I \subset J$ and $J$ is a minimal closed left ideal of $S$, therefore $I=J$, i.e., $J x_{0}=\left\{u_{0}\right\}$. We have shown that for each $x_{0} \in X, J x_{0}$ is a one-point subset of $X$. This implies that $J$ contains but a single mapping, which by our earlier remarks must be a retraction of $X$ onto $F(S)$.

Next, suppose $(X, d)$ is a separable complete metric space and $S$ is equicontinuous. Then $\bar{S}$ is also an equicontinuous semigroup on $X$ (this follows from [11, p. 232]). We will show that $\bar{S}$ is topologically complete, then construct a one-element left ideal of $\bar{S}$ as the intersection of a descending sequence of closed left ideals whose diameters tend to 0 . 
The topology of pointwise convergence on $\bar{S}$ can be metrized by choosing a dense sequence $\left\{p_{n}\right\}$ in $(X, d)$ and defining a metric $\rho$ by

$$
\rho(s, t)=\sum_{i=1}^{\infty} 2^{-i} d\left(s\left(p_{i}\right), t\left(p_{i}\right)\right) /\left[1+d\left(s\left(p_{i}\right), t\left(p_{\imath}\right)\right)\right] .
$$

It is immediate that $(\bar{S}, \rho)$ is complete.

For $u \in F(S)$ and $k$ a positive integer, define

$$
\begin{aligned}
& N_{k}(u)=\{x \in X \mid d(s(x), u) \leqq 1 / k \text { for all } s \in S \text { and } \\
& \quad \text { also for } s=\text { identity on } X\} .
\end{aligned}
$$

We claim

$$
\begin{aligned}
& N_{k}(u) \text { is a closed } \bar{S} \text {-invariant neighborhood of } u \text { with } \\
& \operatorname{diam} N_{k}(u) \leqq 2 / k .
\end{aligned}
$$

Indeed, $N_{k}(u)$ is: closed because each $s \in \bar{S}$ is continuous; $\bar{S}$-invariant because $\bar{S}$ is a semigroup; a neighborhood of $u$ because $\bar{S}$ is equicontinuous and $u \in F(\bar{S})$; of diameter $\leqq 2 / k$ because $d(x, u) \leqq 1 / k$ for all $x \in N_{k}(u)$.

The crucial observation is:

if $J$ is any closed left ideal of $\bar{S}, x \in X$, and $k$ is a positive (5) integer, then there exists a closed left ideal $J^{\prime} \subset J$ and a fixed-point $u$ of $S$ with $J^{\prime} x \subset N_{k}(u)$.

We construct $J^{\prime}$ as follows: First, $J x$ is $\bar{S}$-invariant because $J$ is a left ideal, hence $\mathrm{Cl}(J x)$ is $\bar{S}$-invariant. By $(F P), \mathrm{Cl}(J x)$ contains a fixed point $u$ of $\bar{S}$. In particular the neighborhood $N_{k}(u)$ must intersect $J x$. Put $J^{\prime}=\left\{j \in J \mid j(x) \in N_{k}(u)\right\}$. We have just shown that $J^{\prime}$ is nonempty; $J^{\prime}$ is a closed left ideal of $\bar{S}$ because $J$ is a closed left ideal and $N_{k}(u)$ is closed in $X$. We have proven (5).

Now let $n_{1}, n_{2}, \cdots$ be a sequence of positive integers in which every positive integer appears infinitely often. Inductively define a sequence $\left\{J_{k}\right\}$ of closed left ideals of $\bar{S}$ as follows: $J_{0}=\bar{S}$; having chosen, for some $k \geqq 1$, the closed left ideal $J_{k-1}$, choose $J_{k}$ to be a closed left ideal of $\bar{S}, J_{k} \subset J_{k-1}$, and $u_{k} \in F(S)$, to satisfy

$$
J_{k} p_{n_{k}} \subset N_{k}\left(u_{k}\right) \text {. }
$$

This is possible by (5).

Now fix a positive integer $i$. For infinitely many $k, i=n_{k}$, and for such $k$, (6) implies $J_{k} p_{i} \subset N_{k}\left(u_{k}\right)$. Thus (4) implies

$$
\operatorname{diam} J_{k} p_{i} \leqq 2 / k \text { for infinitely many } k \text {. }
$$

Since the ideals $J_{n}$ are descending, for fixed $i$ the sequence of di- 
ameters of $J_{n} p_{i}$ is nonincreasing. Thus (7) implies $\lim _{n} \operatorname{diam} J_{n} p_{i}=$ 0 for each $i$. It follows from (3) that $\lim _{n} \operatorname{diam} J_{n}=0$.

The sets $J_{n}$ are closed, nonempty, descending, and have $\rho$-diameters tending to 0 in the complete metric space $(\bar{S}, \rho)$, therefore $\bigcap_{n} J_{n}$ consists of a single element $e$. But each $J_{n}$ is a left ideal of $\bar{S}$, hence so is $\bigcap_{n} J_{n}=\{e\}$. By our initial remarks, $e$ must be a retraction of $X$ onto $F(S)$.

Proof of equivalence of $(F P)$ and $(F P)^{\prime}$. First, $(F P)^{\prime}$ implies $(F P)$ because a nonempty closed $S$-invariant set $M$ contains Cl $(S x)$ for each $x \in M$, and hence a fixed point of $S$ if $(F P)^{\prime}$ is satisfied.

Conversely, under either hypothesis (a) or (b), $\mathrm{Cl}(S x)$ is $S$-invariant for each $x \in X$. (In case (a), $\mathrm{Cl}(S x)=S x$ because $S$ compact implies $S x$ compact; in case (b), $S x$ is $S$-invariant and each $s \in S$ is continuous.) If $(F P)$ holds then $\mathrm{Cl}(S x)$ must contain a fixed point of $S$, so $(F P)^{\prime}$ holds.

\section{Examples and remarks.}

Example 1. Some hypothesis such as $(C F P)$ is necessary to guarantee the conclusion of Theorem 2 . We give an example of a bounded separable closed convex $C$ and a nonexpansive $f: C \rightarrow C$ whose fixed point set is not a nonexpansive retract of $C$.

Let $C$ be the closed unit ball in the continuous-function space $C[0,1]$ and let $z:[0,1] \rightarrow[0,1]$ be a continuous function for which $z(t)=1$ for $1 / 2 \leqq t \leqq 1$ but $z(t)<1$ for $0 \leqq t<1 / 2$. Define $f$ by $f(x)(t)=z(t) x(t)$. Obviously $f$ maps $C$ into $C$ and is nonexpansive, and $F(f)=\{x \in C \mid x(t)=0$ for $0 \leqq t \leqq 1 / 2\}$. Nevertheless, there does not exist a nonexpansive retraction of $C$ onto $F(f)$. To see this, let $x_{1}$ denote the constant function $1 / 2$. If $F(f)$ were a nonexpansive retract of $C$, there would exist $y_{1} \in F$ with $\left\|y_{1}-y\right\| \leqq\left\|x_{1}-y\right\|$ for all $y \in F(f)$. But since $y_{1}(1 / 2)=0$, for some $t_{0} \in(1 / 2,1)$ we have $y_{1}\left(t_{0}\right)<1 / 2$. Choose $y \in F(f)$ with $y(t) \geqq 0$ for all $t$ and $y\left(t_{0}\right)=1$. Obviously $\left\|x_{1}-y\right\|=1 / 2$, but $\left\|y_{1}-y\right\| \geqq\left|y_{1}\left(t_{0}\right)-y\left(t_{0}\right)\right|>1 / 2$.

EXAMPLE 2. On the other hand, (CFP) itself is not a necessary condition for $F(f)$ to be a nonexpansive retract of $C$. Consider the set $C$ of the previous example and define $g: C \rightarrow C$ by $g(x)(t)=t \cdot x(t)$. Then $F(g)$ consists of only the zero mapping, and is obviously a nonexpansive retract of $C$; but $\{x \in C \mid x(1)=1\}$ is a bounded separable closed convex $g$-invariant subset of $C$ which does not contain a fixed point of $g$.

REMARK 1. If $F(f) \neq \varnothing$, the nonexpansive retraction $e$ con- 
structed in Theorem 2 can be chosen to satisfy: Every closed convex $f$-invariant subset of $C$ is also $e$-invariant. This is because the proof of Theorem 2 still works if we set

$S=\{s: C \rightarrow C \mid s$ is nonexpansive and every closed convex $f$-invariant subset of $C$ is also $s$-invariant\}.

The existence of a retraction having this additional property is easily seen to be equivalent to $(C F P)$.

REMARK 2. The device of forming a convex linear combination of mappings $r=\sum \lambda_{n} r_{n}$ (not necessarily nonexpansive retractions) and showing $F(r)=\bigcap_{n} F\left(r_{n}\right)$ has been used in [6], [14], and especially [13]. We do not know whether (1) is really needed to prove Lemma 1.

REMARK 3. It is an open question whether the commutativity of $S$ in Theorem 1 can be replaced by the assumption that $S$ is a left reversible semigroup (i.e., that any two right ideals of $S$ intersect). It is interesting to note that if $\mathscr{F}$ is a family of nonempty nonexpansive retracts of $C$ which is linearly ordered by $\supset$, then $S=\{r \mid r$ is a nonexpansive retraction of $C$ onto some $F \in \mathscr{F}\}$ is a left reversible semigroup.

REMARK 4. The relationships among the FPP, the HFPP, and the $C F^{\prime} P P$ are unknown, except for the trivial implication $H F P P \rightarrow$ $C F P P$. This is remarkable, because the most general sufficiency condition is still that of Kirk [12]: $C$ has the FPP if $C$ is weakly compact and has normal structure. Since these properties are inherited by closed convex subsets, $C$ also has the HFPP and the CFPP.

REMARK 5. We have stipulated in Lemma 2 that $C$ is bounded because this is necessary to apply Lemma 1 , but also because it is not clear that a set having the FPP must be bounded.

REMARK 6. The method used to prove Lemma 3 also establishes:

Proposition. If $C$ is locally weakly compact and has the CFPP and $\mathscr{F}$ is a family of nonexpansive retracts of $C$ directed by $\supset$, then $\cap\{F \mid F \in \mathscr{F}\}$ is a nonexpansive retract of $C$.

Cf course, the intersection may be empty. In [6] we proved the proposition under the assumption that each $F \in \mathscr{F}$ is weakly 
closed (but without assuming $C$ has the $C F P P$ ). The distinction is sharp, for it was exactly the uncertainty over the compactness properties of the fixed point set of a nonexpansive mapping which caused such a delay in the generalization of the Belluce-Kirk theorem to infinite families. That uncertainty continues, Lemma 1 notwithstanding.

REMARK 7. It is clear that a nonexpansive retract of $C$ is pathwise connected. Even more is true ([6, Theorem 3]): A nonexpansive retract of $C$ is metrically convex. Thus in Theorem 1 the common fixed-point set $F(S)$ is metrically convex.

REMARK 8. We wish to thank Professor W. A. Kirk for pointing out an oversight in the proof of Lemma 3 in the first version of this paper.

Added in proof. Since this paper was submitted, T. C. Lim has proven the equivalence of normal structure and complete normal structure for weakly convex sets (Characterizations of normal structure, Proc. Amer. Math. Soc., 43 (1974), 313-319). Thus the problem of whether a left reversible semigroup of nonexpansive self-mappings of a weakly compact convex set having normal structure has a fixed point has been settled in the affirmative. We still do not know whether normal structure can be replaced by HFPP.

Also, Lemma 1 is true without the hypothesis that $C$ is bounded. The difference is only technical, involving more stringent restrictions on $\left\{\lambda_{n}\right\}$.

\section{REFERENCES}

1. L. P. Belluce and W. A. Kirk, Fixed-point theorems for families of contraction mappings, Pacific J. Math., 18 (1966), 213-217.

2. - Nonexpansive mappings and fixed-points in Banach spaces, Illinois J. Math., 11 (1967), 474-479.

3. M. S. Brodskii and D. P. Milman, On the center of a convex set, Dokl. Akad. Nauk SSSR (N.S.), 59 (1948), 837-840.

4. F. E. Browder, Nonexpansive nonlinear operators in a Banach space, Proc. Nat. Acad. Sci. U.S.A., 54 (1965), 1041-1044.

5. R. E. Bruck, Jr., Nonexpansive retracts of Banach spaces, Bull. Amer. Math. Soc., 76 (1970), 384-386.

6. - Properties of fixed-point sets of nonexpansive mappings, to appear Trans. Amer. Math. Soc., 179 (1973), 251-262.

7. D. G. DeFigueiredo and L. A. Karlovitz, On the extension of contractions on normed spaces, Nonlinear Functional Analysis, Proc. Symp. in Pure Math., 18 part I, American Math. Soc., Providence, 1970.

8. R. E. DeMarr, Common fixed-points for commuting contraction mappings, Pacific J. Math., 13 (1963), 1139-1141. 
9. R. D. Holmes and A. T. Lau, Asymptotically nonexpansive actions of topological semigroups and fixed points, Bull. London Math. Soc., 3 (1971), 343-347.

10. - Nonexpansive actions of topological semigroups and fixed points, J. London Math. Soc., (2), 5 (1972), 330-336.

11. J. L. Kelly, General Topology, Van Nostrand, Princeton, 1955.

12. W. A. Kirk, A fixed point theorem for mappings which do not increase distances, Amer. Math. Monthly, 72 (1965), 1004-1006.

13. — A fixed point theorem for mappings with a nonexpansive iterate, Proc. Amer. Math. Soc., 29 (1971), 294-298.

14. - On successive approximations for nonexpansive mappings in Banach spaces, Glasgow Math. J., 12 (1971), 6-9.

15. T. C. Lim, A fixed point theorem for families of nonexpansive mappings, to appear Pacific J. Math.

16. T. Mitchell, Fixed points of reversible semigroups of nonexpansive mappings, Kodai Math. Sem. Rep., 22 (1970), 322-323.

17. W. Takahashi, Fixed point theorem for amenable semigroup of nonexpansive mappings, Kodai Math. Sem. Rep., 21 (1969), 383-386.

Received July 6, 1973. Partially supported by NSF grants GP-30221 and GP-38516.

UNIVERSity OF SOUthern California 



\section{PACIFIC JOURNAL OF MATHEMATICS}

\section{EDITORS}

RICHARD ARENS (Managing Editor)

University of California

Los Angeles, California 90024

R. A. Beaumont

University of Washington

Seattle, Washington 98105
J. DugundJI

Department of Mathematics

University of Southern California

Los Angeles, California 90007

D. Gilbarg and J. Milgram

Stanford University

Stanford, California 94305

\section{ASSOCIATE EDITORS}

E. F. BECKENBACH

B. H. NeUMANN

F. WOLF

K. YosHIDA

\section{SUPPORTING INSTITUTIONS}

UNIVERSITY OF BRITISH COLUMBIA

CALIFORNIA INSTITUTE OF TECHNOLOGY

UNIVERSITY OF CALIFORNIA

MONTANA STATE UNIVERSITY

UNIVERSITY OF NEVADA

NEW MEXICO STATE UNIVERSITY

OREGON STATE UNIVERSITY

UNIVERSITY OF OREGON

OSAKA UNIVERSITY
UNIVERSITY OF SOUTHERN CALIFORNIA

STANFORD UNIVERSITY

UNIVERSITY OF TOKYO

UNIVERSITY OF UTAH

WASHINGTON STATE UNIVERSITY

UNIVERSITY OF WASHINGTON

$\stackrel{*}{*} \stackrel{*}{*} \stackrel{*}{*}$ AMERICAN MATHEMATICAL SOCIETY




\section{Pacific Journal of Mathematics}

\section{Vol. 53, No. $1 \quad$ March, 1974}

Martin Bartelt, Strongly unique best approximates to a function on a set, and a finite

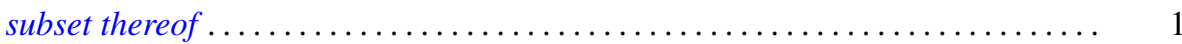

S. J. Bernau, Theorems of Korovkin type for $L_{p}$-spaces $\ldots \ldots \ldots \ldots \ldots \ldots \ldots \ldots \ldots$

S. J. Bernau and Howard E. Lacey, The range of a contractive projection on an

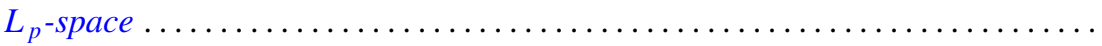

Marilyn Breen, Decomposition theorems for 3-convex subsets of the plane ......... Ronald Elroy Bruck, Jr., A common fixed point theorem for a commuting family of

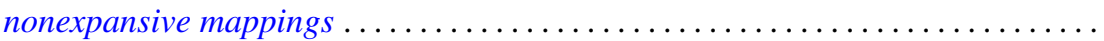

Aiden A. Bruen and J. C. Fisher, Blocking sets and complete $k$-arcs . . . . . . . 73

R. Creighton Buck, Approximation properties of vector valued functions . ......... 85

Mary Rodriguez Embry and Marvin Rosenblum, Spectra, tensor products, and

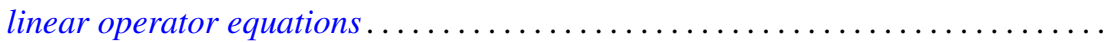

Edward William Formanek, Maximal quotient rings of group rings . . . . . . . . . 109

Barry J. Gardner, Some aspects of T-nilpotence . . . . . . . . . . . . . . . 117

Juan A. Gatica and William A. Kirk, A fixed point theorem for $k$-set-contractions

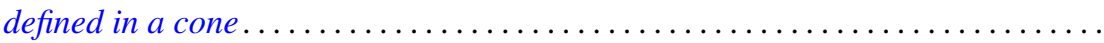

Kenneth R. Goodearl, Localization and splitting in hereditary noetherian prime

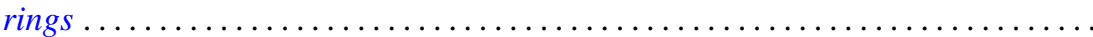

James Victor Herod, Generators for evolution systems with quasi continuous

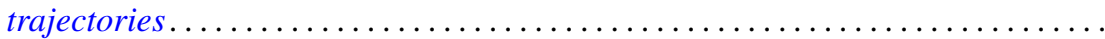

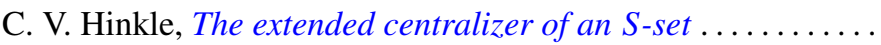

I. Martin (Irving) Isaacs, Lifting Brauer characters of p-solvable groups . . .

Bruce R. Johnson, Generalized Lerch zeta function ...........

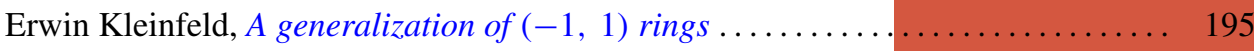

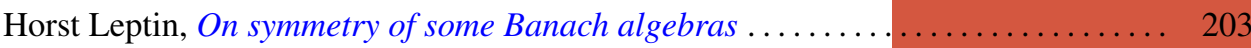

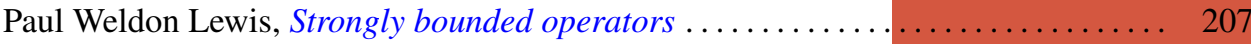

Arthur Larry Lieberman, Spectral distribution of the sum of self-adjoint

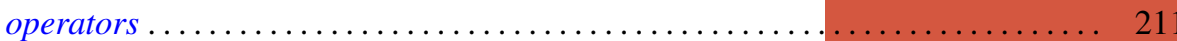

I. J. Maddox and Michael A. L. Willey, Continuous operators on paranormed spaces and matrix transformations

James Dolan Reid, On rings on groups ........................... 229

Richard Miles Schori and James Edward West, Hyperspaces of graphs are Hilbert cubes.

William H. Specht, A factorization theorem for p-constrained groups ...

Robert L Thele, Iterative techniques for approximation of fixed points of certain nonlinear mappings in Banach spaces ...............

Tim Eden Traynor, An elementary proof of the lifting theorem

Charles Irvin Vinsonhaler and William Jennings Wickless, Completely decomposable groups which admit only nilpotent multiplications .

Raymond O’Neil Wells, Jr, Comparison of de Rham and Dolbeault cohomology for

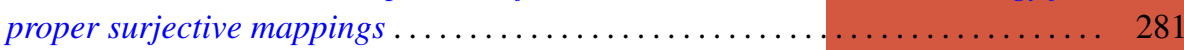

David Lee Wright, The non-minimality of induced central representations . . . . . 301 